Canad. Math. Bull. Vol. 23 (3), 1980

\title{
LEAST POSITIVE RESIDUES AND THE QUADRATIC CHARACTER OF TWO
}

\author{
BY \\ KENNETH H. ROSEN
}

Let $r_{j}^{(t)}$ be the least positive residue modulo $2^{t} k$ of $(2 j-1) h$. Define $u_{t}$ to be the number of $r_{j}^{(t)}$ with $1 \leq j \leq 2^{t-2} k$ such that $2^{t-1} k<r_{j}^{(t)}<2^{t} k$. At the Special Session in Combinatorial Number Theory at the 1977 Summer AMS Meeting Szekeres [2] asked for a simple proof that if $(h, 2 k)=1$, then

$$
u_{4} \equiv\left\{\begin{array}{lllll}
0 & (\bmod 4) & \text { if } & h \equiv \pm 1 & (\bmod 8) \\
2 & (\bmod 4) & \text { if } & h \equiv \pm 3 & (\bmod 8)
\end{array}\right.
$$

Here a simple proof will be given for the following equivalent result.

THEOREM 1. If $(h, 2 k)=1$, then if $t \geq 4$

$$
u_{t} \equiv\left\{\begin{array}{lllll}
0 & (\bmod 4) & \text { if } & h \equiv \pm 1 & (\bmod 8) \\
2 & (\bmod 4) & \text { if } & h \equiv \pm 3 & (\bmod 8)
\end{array}\right.
$$

To prove Theorem 1, one first expresses $u_{t}$ as a sum.

LemMA 2. If $(h, 2 k)=1$, then $u_{t}=S_{1}-2 S_{2}$, where

$$
S_{1}=\sum_{j=1}^{2^{t-2 k}}\left[\frac{(2 j-1) h}{2^{t-1} k}\right] \text { and } S_{2}=\sum_{j=1}^{2^{t-2 k}}\left[\frac{(2 j-1) h}{2^{t} k}\right] \text {. }
$$

Proof. Lemma 2 follows from the fact that the difference

$$
\left[\frac{(2 j-1) h}{2^{t-1} k}\right]-2\left[\frac{(2 j-1) h}{2^{t} k}\right]
$$

is zero when $0<r_{j}^{(t)}<2^{t-1} k$ and is one when $2^{t-1} k<r_{j}^{(t)}<2^{t} k$.

The following easily verified identities are useful.

$$
\begin{aligned}
& S_{1}=\sum_{j=1}^{2^{t}-1 k}\left[\frac{j h}{2^{t-1} k}\right]-\sum_{j=1}^{2 t-2 k}\left[\frac{j h}{2^{t-2} k}\right] \text { and } \\
& S_{2}=\sum_{j=1}^{2 t-1 k}\left[\frac{j h}{2^{t} k}\right]-\sum_{j=1}^{2^{t-2 k}}\left[\frac{j h}{2^{t-1} k}\right] .
\end{aligned}
$$

Received by the editors March 29, 1978 and in revised form, November 23, 1978 and February $1,1979$. 
To evaluate $S_{1}$ one uses

LEMMA 3. If $(m, h)=1$, then

$$
\sum_{j=1}^{m}\left[\frac{j h}{m}\right]=h+\frac{(h-1)(m-1)}{2} .
$$

Proof. Let $\{x\}=x-[x]$. Then

$$
\sum_{j=1}^{m-1}\left[\frac{j h}{m}\right]=\sum_{j=1}^{m-1}\left(\frac{j h}{m}-\left\{\frac{j h}{m}\right\}\right)=\frac{h}{m} \frac{m(m-1)}{2}-\sum_{\ell=1}^{m-1} \frac{\ell}{m}=\frac{(h-1)(m-1)}{2} .
$$

The second equality is true because as $j$ runs through a full set of residues mod $m$, so does $j h$. Lemma 3 follows by addition of $h$ to the first and last members of this string of equalities.

Applying Lemma 3 to (1), one concludes that

$$
S_{1}=\frac{1}{2}(h-1) 2^{t-2} k
$$

To deal with $S_{2}$, it is first necessary to prove

LEMMA 4. If $(h, 2 k)=1$ and if $c$ is a nonnegative integer, then

$$
\sum_{j=1}^{2 c k}\left[\frac{j h}{2^{c+1} k}\right]=-\sum_{j=1}^{(h-1) / 2}\left[\frac{2^{c+1} k j}{h}\right]+2^{c} k\left(\frac{h-1}{2}\right)
$$

Proof. Count the number of terms of the sum on the left hand side of (4) that equal a fixed number $n$. If $1 \leqq n \leqq(h-3) / 2$ then $n$ occurs exactly

$$
\left[\frac{2^{c+1} k(n+1)}{h}\right]-\left[\frac{2^{c+1} k n}{h}\right]
$$

times. The number $(h-1) / 2$ occurs exactly

$$
2^{c} k-\left[\frac{2^{c+1} k(h-1)}{2 h}\right]
$$

times. Hence

$$
\begin{aligned}
\sum_{j=1}^{2^{c k}}\left[\frac{j h}{2^{c+1} k}\right]= & \sum_{n=1}^{(h-3) / 2} n\left(\left[\frac{2^{c+1}(n+1) k}{h}\right]-\left[\frac{2^{c+1} n k}{h}\right]\right) \\
& +\frac{h-1}{2}\left(2^{c} k-\left[\frac{2^{c+1} k(h-1)}{2 h}\right]\right) \\
= & -\sum_{j=1}^{(h-1) / 2}\left[\frac{2^{c+1} k j}{h}\right]+2^{c} k\left(\frac{h-1}{2}\right) .
\end{aligned}
$$


From (2) and Lemma 4, one obtains

$$
S_{2}=\left(\frac{h-1}{2}\right) 2^{t-2} k+\sum_{j=1}^{(h-1) / 2}\left[\frac{2^{t-1} k j}{h}\right]-\sum_{j=1}^{(h-1) / 2}\left[\frac{2^{t} k j}{h}\right] .
$$

The following classical result which is proved in Bachmann [1] relates the sums on the right hand side of (5) to the Jacobi symbol.

LEMMA 5. $(h, 2 q)=1$, then

$$
\sum_{j=1}^{(h-1) / 2}\left[\frac{2 q j}{h}\right] \equiv \frac{1}{2}\left(\left(\frac{q}{h}\right)-1\right)(\bmod 2)
$$

where $(q / h)$ is the Jacobi symbol.

Consequently, from (3), (5) and Lemma 5, one obtains

$$
u_{t}=S_{1}-2 S_{2}=-\frac{1}{2}(h-1) 2^{t-2} k+\left[1-\left(\frac{2}{h}\right)\right](\bmod 4) .
$$

Theorem 1 now follows from (6), by noting that

$$
\left(\frac{2}{h}\right)=\left\{\begin{array}{rlll}
1 & \text { if } & h \equiv \pm 1 \quad(\bmod 8) \\
-1 & \text { if } & h \equiv \pm 3 \quad(\bmod 8)
\end{array}\right.
$$

When $t-2$ or $t=3$ note that from (6) one can prove

THEOREM 6. If $(h, 2 k)=1$, then

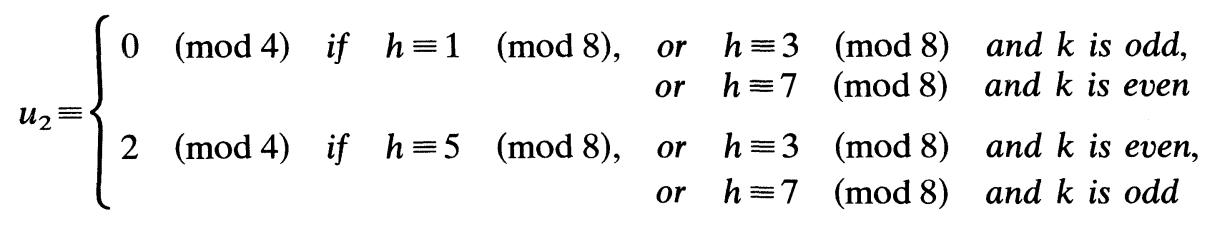

and

$$
u_{2} \equiv\left\{\begin{array}{lllll}
0 & (\bmod 4) & \text { if } & h \equiv 1 & (\bmod 8) \\
3 k+2 & (\bmod 4) & \text { if } & h \equiv 3 & (\bmod 8) \\
2 k+2 & (\bmod 4) & \text { if } & h \equiv 5 & (\bmod 8) \\
k & (\bmod 4) & \text { if } & h \equiv 7 & (\bmod 8)
\end{array}\right.
$$

Note ADDED ON SEPTEMBer 20, 1978. A different proof of Theorem 1 has been given by G. Szekeres and B. Richmond in their interesting paper, The Taylor Coefficients of Certain Infinite Products, Acta Sci. Math. Szeged 40 (1978) 347-369 as a commemorative article for Professor Turán. Szekeres has remarked, and the author agrees, that it would be desirable to prove Theorem 1 as a consequence of Gauss' Lemma. 
AcKnowledgement. The author would like to thank the referee and Prof. Ronald Evans for their helpful comments.

\section{REFERENCES}

1. P. Bachmann, Die Elemente der Zahlentheorie, Teubner, Leipzig, 1892, (reprint: Chelsea, New York, 1968), p. 144-148.

2. C. Long, Problem List, Combinatorial Number Theory, Notices of the American Mathematical Society, 25 (1978), p. 145.

\section{Department of Mathematics \\ UNIVERSITY OF COLORADO \\ BOUlder, COLORADO 80309 USA}

Author's Current AdDress:

DepartMent OF MATHEMATICS

The UnIVERsity OF MAINE

OROND, MAINE, 04469 USA 